\title{
An Extremely Efficient and Compact Long- Wavelength Cut-Off Plasmonic Filter based on Stepped Impedance Resonators
}

\author{
Seyed Morteza Ebadi*, Student Member, IEEE, and Jonas Örtegren \\ Department of Natural Sciences, Mid Sweden University, SE-85170 Sundsvall, Sweden \\ *seyed-morteza.ebadi@miun.se
}

\begin{abstract}
We report the design and numerical results of a miniaturized and efficient plasmonic cut-off filter that is based on stepped impedance resonators and operates at telecom wavelengths.
\end{abstract}

Keywords-; Plasmonics; optical filters; photonic integrated circuits, stepped impedance resonators (SIRs).

\section{INTRODUCTION}

Plasmonic technology is regarded as one of the most promising platforms that can overcome the diffraction limit of light, and enable light confinement at subwavelength scales. Surface plasmon polaritons (SPPs), emerge from the interaction of surface electromagnetic waves and free electron oscillations at a metal-dielectric interface, resulting in guidance and extreme mode confinement of the electromagnetic wave. Plasmonics would offer the capability to integrate CMOS-based electronics and photonic circuits on the same chip, which significantly contributes to miniaturizing the size of components and enables a small foot-print and an energy-efficient solution for development of photonic integrated circuits (PICs) $[1,2]$. As a consequence, a variety of optical components based on plasmonics such as highspeed modulators [3], perfect light absorbers [4], and so on were suggested. Indeed, optical filter, whose ability to pass a specific wavelength while blocking others are regarded as an essential part of many systems, including dispersion compensation, fluorescent microscopy, color match imaging, and many more.

\section{Device Structure AND Discussion}

We suggest a novel ultrasmall scale long wavelength cut-off filter based on stepped impedance resonators (SIRs) embedded in a metal-insulator-metal (MIM) plasmonic waveguide that functions at optical channels. The proposed filter is demonstrated in Fig. 1. (a), and consists of two layers of silver, whose complex dielectric constant is taken from experimental data by Johnson and Christy [5]. The insulator layer that is located between the metallic layers is chosen to be air. The fundamental TM mode of the plasmonic waveguide is excited through a dipole source. The grid sizes are chosen to be $5 \mathrm{~nm} \times 5 \mathrm{~nm}$ along the $\mathrm{x}$ and $\mathrm{y}$ directions, respectively. Two power monitors $\mathrm{P}$ and $\mathrm{Q}$, are set to record the incident and transmitted power in the structure, and that $\mathrm{T}=$ Pout/Pin is defined as the transmittance of the device. The simulation is carried out by using a full-wave EM tool, CST MWS, based on finite element method (FEM) [6]. The transmittance and reflectance profile of the proposed cut-off filter is demonstrated in Fig. 1. (b). One can define the cut-off wavelength to where the transmittance reaches to $1 \%$ [7], which is at the wavelength $1289 \mathrm{~nm}$. It should be mentioned that the structural parameters are optimized to make the structure symmetric to make it easier for experimental realization of the device.
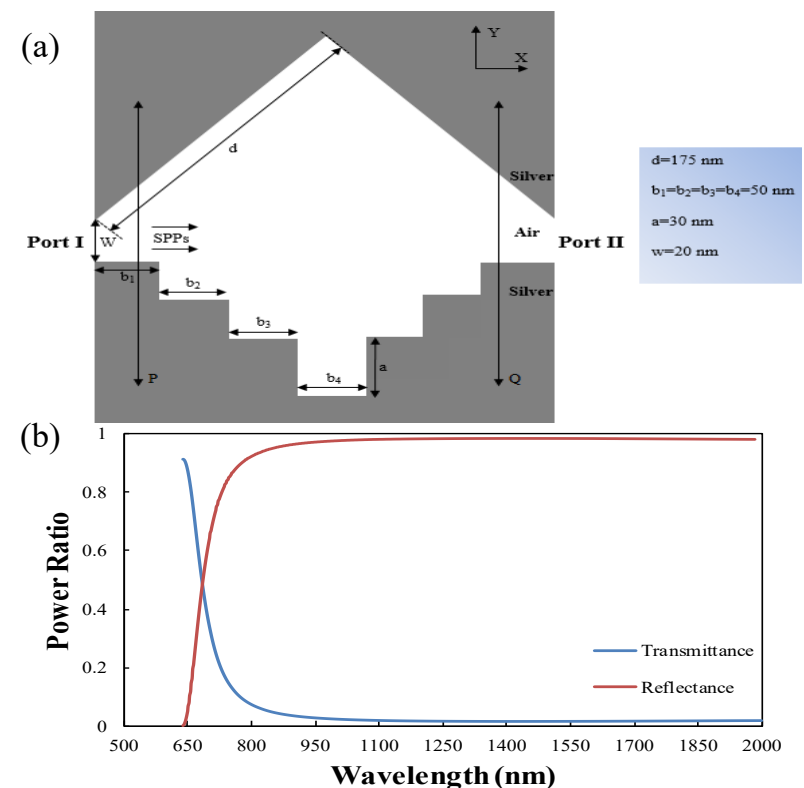

Fig. 1. (a) Schematic configuration of the suggested long wavelength cutoff filter. (b) transmission and reflection spectra of the filter with values that shown in Fig. 1. (a).

\section{CONCLUSION}

In conclusion, a chip-scale and highly-efficient plasmonic long wavelength cut-off filter at telecom wavelength is proposed and numerically investigated. The new structure compared to recent studies $[6,7]$, provides a smaller foot-print and may find applications in on-chip integration and in wireless optical communication systems.

\section{REFERENCES}

[1] Gramotnev, Dmitri K., and Sergey I. Bozhevolnyi. "Plasmonics beyond the diffraction limit." Nature photonics 4.2 (2010): 83-91.

[2] Koch, Ueli, et al. "A monolithic bipolar CMOS electronic-plasmonic high-speed transmitter." Nature Electronics 3.6 (2020): 338-345.

[3] Hössbacher, Claudia, et al. "Plasmonic modulator with $>170 \mathrm{GHz}$ bandwidth demonstrated at 100 GBd NRZ." Optics Express 25.3 (2017): 1762-1768.

[4] Gao, Huixuan, et al. "Plasmonic broadband perfect absorber for visible light solar cells application." Plasmonics 15.2 (2020): 573-580.

[5] Johnson, Peter B., and Christy, R. W. "Optical constants of the noble metals." Physical review B 6.12 (1972): 4370.

[6] Ebadi, S.M, et al. "A Multipurpose and Highly-Compact Plasmonic Filter Based on Metal-Insulator-Metal Waveguides." IEEE Photonics Journal 12.3 (2020): 1-9.

[7] Ebadi, Seyed Morteza, et al. "A new plasmonic long wavelength cutoff filter based on triangular arrays in a MIM waveguide." 2015 Photonics North. IEEE, 2015 